\title{
DIAGNÓSTICO DA ARBORIZAÇÃO DA ÁREA CENTRAL DA CIDADE DE SANTO CRISTO - RS
}

\author{
Márcia Cristina Gerhardt ${ }^{1}$, Nilvane Ghellar Muller $^{2}$, Silvia Raquel Scherer Wolski ${ }^{3}$
}

(recebido em 05.10.2010 e aceito para publicação em 15.03.2011)

\section{RESUMO}

Realizou-se o diagnóstico quali-quantitativo da arborização da área central da cidade de Santo Cristo - RS. Nessa área central, previamente demarcada em mapa da cidade, foi realizada uma amostragem, totalizando 28 ruas. Os dados foram coletados em formulário padronizado, que continha informações sobre data de coleta, nome da rua, nome vulgar das espécies, circunferência à altura do peito, altura, fitossanidade da árvore e de poda e espaçamento e afastamento entre árvores. Nas 28 ruas inventariadas, foram encontradas 3403 plantas arbóreas, pertencentes a 63 espécies, sendo uma delas, Cinnamomum zeylanicum Blume, responsável por quase $30 \%$ dos indivíduos levantados. O predomínio de espécies exóticas foi bem significativo na composição da arborização, o que não é recomendado. Das condições fitossanitárias dos indivíduos pesquisados, houve uma predominância de 79,43 \% danificados, decorrente da má utilização da poda, da concentração de espécies exóticas e da falta de diversidade entre elas. Diante deste estudo, é necessário realizar um planejamento dessa área central, respeitando as limitações de cada espécie, diversificando a arborização e fazendo o devido manejo desses indivíduos, afim de evitar mais conflitos e usufruir assim dos seus benefícios.

Palavras-chave: arborização urbana, planejamento urbano.

\footnotetext{
${ }^{1}$ Acadêmica do Curso de Ciências Biológicas da Universidade Regional Integrada do Alto Uruguai e das Missões, Campus de Santo Ângelo,RS. Email:mgerbio@urisan.tche.br.

2 Professora, Dr ${ }^{\mathrm{a}}$., da Universidade Regional Integrada do Alto Uruguai e das Missões - Campus de Santo Ângelo RS.

${ }^{3}$ Engenheira Florestal, M. Sc., da Secretaria Estadual da Agricultura - Santo Ângelo - RS.
}

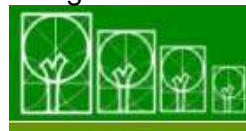

S $\cdot \mathbf{B} \cdot \mathbf{A} \cdot \mathbf{U}$ Soc. Bras. de Arborização Urbana 


\title{
URBAN FOREST DIAGNOSIS IN DOWNTOWN AREA OF CITY OF SANTO CRISTO, RS, BRAZIL
}

\begin{abstract}
In this study was done a quail-quantitative diagnosis of urban forest in downtown area of city of Santo Cristo, RS. A sampling of 28 streets was performed. Data were collected in a standardized form, which contained information about date of collection, name of street, common name of species, circumference at breast height, height, tree phytosanity, spacing and distance between trees. In 28 streets scheduled 3403 woody plants were found, belonging to 63 species, one of which, Cinnamomum zeylanicum Blume, was responsible for almost $30 \%$ of surveyed individuals. Dominance of exotic species was quite significant in composition of urban trees and this is not recommended. Considering phytosanity conditions of individuals surveyed, there was a prevalence of $79.43 \%$ damaged, resulting from misuse of pruning, concentration of exotic species and lack of diversity among them. Facing this study, it is necessary perform a planning to downtown area, within the limits of each species, diversifying the urban forest and making the proper management of individuals in order to avoid further conflicts and therefore enjoy benefits.
\end{abstract}

Keywords: urban forest, urban planning.

\section{INTRODUÇÃO}

A arborização exerce papel de vital importância para a qualidade de vida nos centros urbanos. Por suas múltiplas funções, a árvore urbana atua diretamente sobre o clima, a qualidade do ar, o nível de ruídos e sobre a paisagem, além de constituir refúgio indispensável à fauna remanescente nas cidades.

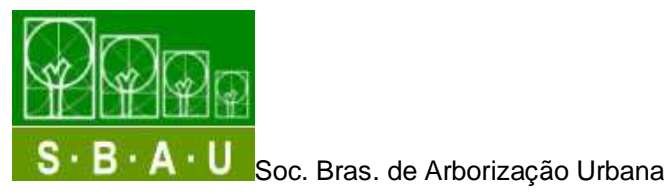

REVSBAU, Piracicaba - SP, v.6, n.1, p.69-84, 2011 
Arborizar uma cidade não significa apenas plantar árvores em ruas, jardins e praças. A arborização deve atingir objetivos de ornamentação, de melhoria micro-climática e de diminuição da poluição, assim seu planejamento deve considerar aspectos ambientais, culturais e históricos. (MILANO, 1984).

Devido à falta de planejamento da arborização, indispensável para o desenvolvimento urbano, bem como da ausência de planejamento de outros elementos que compõem o cenário urbano (postes de iluminação, fiações, telefones públicos) existe, na grande maioria das cidades, um conflito entre esses elementos, uma vez que convivem em constante desarmonia.

A cidade de Santo Cristo apresenta uma área central com árvores distribuídas sem planejamento, que causam vários problemas característicos de muitas áreas urbanas, nas quais se sabe da grande dificuldade de se implantar o verde, principalmente, se conciliado à presença de equipamentos urbanos (SOARES, 1998). Por se tratar de uma cidade em crescente desenvolvimento, o planejamento da arborização é especialmente importante para potencializar os benefícios e evitar a ocorrência de conflitos.

Para planejar é necessário, primeiramente, conhecer. O conhecimento da arborização urbana é possível através de um diagnóstico quali-quantitativo deve ter como objetivo conhecer as espécies que compõem a arborização, os espaçamentos utilizados, a ocorrência de podas e o estado fitossanitário das árvores.

Com o objetivo de conhecer a situação da arborização da área central da cidade de Santo Cristo, o presente trabalho realizou o levantamento da arborização dessa área buscando, desta forma, contribuir para o planejamento urbano e ambiental a partir das análises geradas.

\section{MATERIAIS E MÉTODOS}

O estudo foi realizado na área central da cidade de Santo Cristo, que está situada na fronteira noroeste do Estado do Rio Grande do Sul. Localiza-se na latitude de 27049'46"sul e em longitude 54³9'46" oeste, estando em altitude de 283 metros. Dista 516 km da capital, Porto Alegre. A temperatura média anual é de $19^{\circ} \mathrm{C}$ e a pluviosidade média é de $1600 \mathrm{~mm} / \mathrm{ano}$. Possui uma população urbana de 7.284 habitantes.(IBGE, 2006).

O levantamento quali-quantitativo foi realizado através de uma amostra da arborização urbana das vias públicas em uma área de aproximadamente 30 quadras do centro da cidade de

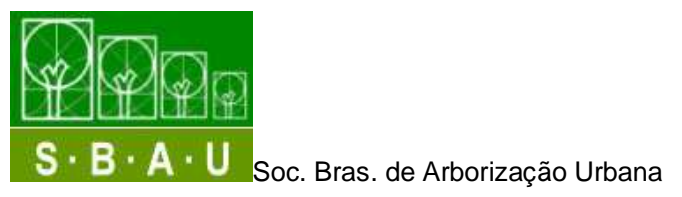

REVSBAU, Piracicaba - SP, v.6, n.1, p.69-84 2011 
Santo Cristo. Os itens levantados foram os seguintes: (a) número da árvore a ser inventariada; (b) nome popular; (c) CAP (circunferência à altura do peito tomada a 1,30 m do solo) em cm; (d) altura aproximada (de forma visual, comparativamente parâmetros de alturas como postes e casas); (e) situação das raízes nas calçadas; (f) estado fitossanitário das árvores; (g) espaçamento e (h) afastamento entre árvores; (i) poda;

A descrição das espécies foi realizada com base na literatura especializada sobre o assunto (BACKES e IRGANG, 2002) e coleta de dados em campo, sendo fotografadas as espécies existentes e os dados foram inseridos numa planilha do software Microsoft Office Excel 2003. Os dados foram levantados durante mês de fevereiro de 2007.

\section{RESULTADOS E DISCUSSÃo}

O levantamento realizado identificou um total de 3403 árvores, distribuídas na área central delimitada da cidade de Santo Cristo. Nestas, foram identificadas 63 espécies arbóreas, destacando-se a espécie Cinnamomum zeylanicum Blume. Essa espécie exótica, originária do Sri Lanka, de porte médio, casca pálida e sem pêlos, folhas simples, flores pequenas brancoamareladas e de crescimento rápido, teve uma participação de aproximadamente $30 \%$ na composição da arborização (BACKES e IRGANG, 2002), conforme Tabela 1.

TABELA 1 - Relação das famílias, espécies, número de indivíduos e da freqüência dos exemplares vegetais encontrados na arborização das vias públicas da área central de Santo Cristo, RS.

TABLE 1 - Relation of families, species, number of individuals and frequency of plants encountered in urban forest of central area of Santo Cristo, RS.

\begin{tabular}{|l|l|l|c|c|}
\hline Família & Nome científico & Nome vulgar & $\begin{array}{c}\text { No } \\
\text { indivíduos }\end{array}$ & $\begin{array}{c}\text { Freqüência } \\
(\%)\end{array}$ \\
\hline Lauraceae & $\begin{array}{l}\text { Cinnamomum zeylanicum } \\
\text { Blume }\end{array}$ & Canelinha & 993 & 29,18 \\
\hline Lythraceae & Lagerstroemia indica Blanco & Extremosa & 607 & 17,84 \\
\hline Oleaceae & Ligustrum japonicum Thunb & Ligustro & 294 & 8,64 \\
\hline Rutaceae & Murraya Paniculata .Jack & Falsa-murta & 218 & 6,41 \\
\hline Moraceae & Ficus benjamina.L & Ficus & 140 & 4,11 \\
\hline
\end{tabular}

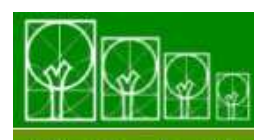

$\mathbf{S} \cdot \mathbf{B} \cdot \mathbf{A} \cdot \mathbf{U}$ Soc. Bras. de Arborização Urbana 
TABELA 1 - (continuação) Relação das famílias, espécies, número de indivíduos e da freqüência dos exemplares vegetais encontrados na arborização das vias públicas da área central de Santo Cristo, RS.

TABLE 1 - (continuation) Relation of families, species, number of individuals and frequency of plants encountered in urban forest of central area of Santo Cristo, RS.

\begin{tabular}{|c|c|c|c|c|}
\hline Palmae & $\begin{array}{l}\text { Archontophoenix alexandrae } \\
\text { (F. Muell.) H. Wendl. \& Drude }\end{array}$ & Palmeira-real & 129 & 3,79 \\
\hline Anacardiaceae & Mangifera indica.L & Mangueira & 126 & 3,70 \\
\hline $\begin{array}{l}\text { Fabaceae - } \\
\text { Caesalpiniodeae }\end{array}$ & Caesalpinia peltophoroides .L & Sibipiruna & 131 & 2,94 \\
\hline Bignoniaceae & $\begin{array}{l}\text { Tabebuia chrysotricha } \\
\text { Standl. }\end{array}$ & Ipê & 74 & 2,17 \\
\hline Proteaceae & $\begin{array}{l}\text { Grevillea robusta A. Cunn. ex } \\
\text { R. Br. }\end{array}$ & Grevilea & 66 & 1,94 \\
\hline Cupressaceae & Thuja plicata Donn ex D. Don & Tuia & 65 & 1,91 \\
\hline Bignoniaceae & $\begin{array}{l}\text { Tabebuia avallanedae (Vell.) } \\
\text { Toledo }\end{array}$ & Ipê roxo & 65 & 1,91 \\
\hline Bignoniaceae & $\begin{array}{l}\text { Spathodea campanulata P. } \\
\text { Beauv. }\end{array}$ & Espatódea & 56 & 1,65 \\
\hline Malvaceae & Hibiscus rosa-sinensis. $L$ & Hibiscus & 24 & 0,70 \\
\hline Rosaceae & Prunus domestica.L & Ameixa & 24 & 0,70 \\
\hline Rutaceae & Citrus limon. (L.) Burm.f. & Limoeiro & 21 & 0,61 \\
\hline Myrtaceae & Psidium cattleyanum. Sabine & Araçá & 21 & 0,61 \\
\hline Fabaceae & Inga SP.Duque & Ingá & 20 & 0,59 \\
\hline $\begin{array}{l}\text { Fabaceae - } \\
\text { Faboideae }\end{array}$ & Tipuana tipu. (Benth.) Kuntze & Tipuana & 20 & 0,59 \\
\hline $\begin{array}{l}\text { Fabaceae - } \\
\text { Mimosoideae }\end{array}$ & $\begin{array}{l}\text { Enterolobium } \\
\text { contortisiliquum. (Vell.) } \\
\text { Hauman }\end{array}$ & Timbaúva & 17 & 0,49 \\
\hline Myrtaceae & Eugenia uniflora. L. & Pitangueira & 16 & 0,47 \\
\hline Juglandaceae & Carya illinoensis $\mathrm{K}$. & $\begin{array}{l}\text { Nogueira } \\
\text { pecã }\end{array}$ & 16 & 0,47 \\
\hline Cupressaceae & $\begin{array}{l}\text { Chamaecyparis obtusa } \\
\text { (Siebold \& Zucc.) Endl. }\end{array}$ & $\begin{array}{l}\text { Cipreste } \\
\text { dourado }\end{array}$ & 15 & 0,45 \\
\hline $\begin{array}{l}\text { Fabaceae - } \\
\text { Cercideae }\end{array}$ & Bauhinia SP Blanco. & Pata-de-vaca & 14 & 0,41 \\
\hline Verbenaceae & Duranta repens L. & $\begin{array}{l}\text { Pingo de } \\
\text { ouro }\end{array}$ & 13 & 0,38 \\
\hline Meliaceae & Cedrela fissilis Vell. & Cedro & 12 & 0,35 \\
\hline Anacardiaceae & Schinus molle L. & $\begin{array}{l}\text { Aroeira } \\
\text { piriquita }\end{array}$ & 12 & 0,35 \\
\hline Lauraceae & Cordia trichotoma (Vell.) & Louro & 12 & 0,35 \\
\hline
\end{tabular}

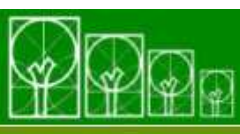

$\mathbf{S} \cdot \mathbf{B} \cdot \mathbf{A} \cdot \mathbf{U}$ Soc. Bras. de Arborização Urbana 
TABELA 1 - (continuação) Relação das famílias, espécies, número de indivíduos e da freqüência dos exemplares vegetais encontrados na arborização das vias públicas da área central de Santo Cristo, RS.

TABLE 1 - (continuation) Relation of families, species, number of individuals and frequency of plants encountered in urban forest of central area of Santo Cristo, RS.

\begin{tabular}{|c|c|c|c|c|}
\hline Lauraceae & $\begin{array}{l}\text { Cinnamomum camphora (L.) } \\
\text { J. Pres }\end{array}$ & Canforeira & 12 & 0,35 \\
\hline Arecaceae & $\begin{array}{l}\text { Butia capitata (Martius) } \\
\text { Beccari }\end{array}$ & Butiá & 11 & 0,32 \\
\hline Myrtaceae & Psidium guajava L. & Goiaba & 11 & 0,32 \\
\hline Boraginaceae & Patagonula americana L. & Guajuvira & 11 & 0,32 \\
\hline $\begin{array}{l}\text { Fabaceae - } \\
\text { Caesalpiniodeae } \\
\text { Arecaceae }\end{array}$ & $\begin{array}{l}\text { Senna multijuga } \\
\text { (Rich.) Irwin et Barn. } \\
\text { Cocos nucifera }\end{array}$ & $\begin{array}{l}\text { Chuva de } \\
\text { ouro } \\
\text { Coqueiro }\end{array}$ & $\begin{array}{l}11 \\
10\end{array}$ & $\begin{array}{l}0,32 \\
0,29\end{array}$ \\
\hline $\begin{array}{l}\text { Leguminosae- } \\
\text { Caesalpiniodeae }\end{array}$ & $\begin{array}{l}\text { Senna macranthera } \\
\text { (Collad.) Irwin et Barn. }\end{array}$ & Manduirana & 08 & 0,23 \\
\hline Rutaceae & Citrus SSPL. & Bergamoteira & 08 & 0,23 \\
\hline Platanaceae & Platanus occidentalis L. & Plátano & 07 & 0,21 \\
\hline Rutaceae & Citrus sinensis ( L.) Osbeck. & Laranjeira & 07 & 0,20 \\
\hline Meliaceae & Melia azeradach L. & Cinamomo & 06 & 0,18 \\
\hline $\begin{array}{l}\text { Leguminosae- } \\
\text { Caesalpiniodeae }\end{array}$ & Delonix regia Stehlé. & Flamboyant & 06 & 0,18 \\
\hline Mimosoideae & Calliandra brevipes Benth. & Esponjinha & 06 & 0,18 \\
\hline $\begin{array}{l}\text { Não identificada } \\
3\end{array}$ & & & 06 & 0,18 \\
\hline Rhamnaceae & Hovenia dulcis Thunb. & $\begin{array}{l}\text { Uva do } \\
\text { Japão }\end{array}$ & 05 & 0,15 \\
\hline Myrtaceae & $\begin{array}{l}\text { Syzygium malaccense (L.) } \\
\text { Merr. \& L.M. Perry }\end{array}$ & Jambolão & 05 & 0,15 \\
\hline Annonaceae & Rollinia salicifolia Schltdl. & Araticum & 05 & 0,15 \\
\hline Ebenaceae & Diospyros kaki Makino. & Caqui & 04 & 0,12 \\
\hline Araucariaceae & $\begin{array}{l}\text { Araucaria angustifolia } \\
\text { (Bertol.) Kuntze. }\end{array}$ & Araucária & 04 & 0,12 \\
\hline Myrtaceae & $\begin{array}{l}\text { Campomanesia xanthocarpa } \\
\text { Berg. }\end{array}$ & Gabiroba & 04 & 0,12 \\
\hline $\begin{array}{l}\text { Não identificada } \\
2\end{array}$ & & & 04 & 0,12 \\
\hline $\begin{array}{l}\text { Fabaceae - } \\
\text { Caesalpiniodeae }\end{array}$ & $\begin{array}{l}\text { Caesalpinia férrea } \\
\text { Mart. }\end{array}$ & Pau-ferro & 03 & 0,09 \\
\hline $\begin{array}{l}\text { Leguminosae- } \\
\text { Caesalpinoideae }\end{array}$ & $\begin{array}{l}\text { Holocalyx balansae } \\
\text { Mich. }\end{array}$ & Alecrim & 03 & 0,09 \\
\hline Lauraceae & $\begin{array}{l}\text { Persea americana (L.O. } \\
\text { Williams). }\end{array}$ & Abacateiro & 03 & 0,08 \\
\hline Apocynaceae & Nerium oleander $\mathrm{L}$. & Espirradeira & 02 & 0,06 \\
\hline
\end{tabular}

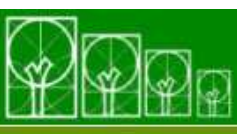

$\mathbf{S} \cdot \mathbf{B} \cdot \mathbf{A} \cdot \mathbf{U}$ Soc. Bras. de Arborização Urbana 
TABELA 1 - (continuação) Relação das famílias, espécies, número de indivíduos e da freqüência dos exemplares vegetais encontrados na arborização das vias públicas da área central de Santo Cristo, RS.

TABLE 1 - (continuation) Relation of families, species, number of individuals and frequency of plants encountered in urban forest of central area of Santo Cristo, RS.

\begin{tabular}{|l|l|l|c|c|}
\hline Bombacaceae & Chorisia speciosa A. St.-Hil. & Paineira & 02 & 0,06 \\
\hline $\begin{array}{l}\text { Fabaceae - } \\
\text { Caesalpiniodeae }\end{array}$ & $\begin{array}{l}\text { Schizolobium parahyba } \\
\text { (Vell.) S.F. Blake) }\end{array}$ & Guapuruvú & 02 & 0,06 \\
\hline Myrtaceae & $\begin{array}{l}\text { Myrciaria cauliflora (Mart.) O. } \\
\text { Berg. }\end{array}$ & Jabuticaba & 02 & 0,06 \\
\hline Caricaceae & Carica papaya L. & Mamoeiro & 02 & 0,06 \\
\hline Salicaceae & Salix babilónica L. & Chorão & 02 & 0,06 \\
\hline Bignoniaceae & $\begin{array}{l}\text { Jacaranda copaia (Aubl.) D. } \\
\text { Don. }\end{array}$ & Caroba & 02 & 0,06 \\
\hline $\begin{array}{l}\text { Fabaceae - } \\
\text { Faboideae }\end{array}$ & $\begin{array}{l}\text { Dalbergia brasiliensis } \\
\text { Vogel. }\end{array}$ & Jacarandá & 01 & 0,03 \\
\hline Myrtaceae & $\begin{array}{l}\text { Callistenon viminalis G. Don } \\
\text { ex Loud. }\end{array}$ & $\begin{array}{l}\text { Escova-de- } \\
\text { garrafa }\end{array}$ & 01 & 0,03 \\
\hline $\begin{array}{l}\text { Fabaceae - } \\
\text { Caesalpiniodeae } \\
\text { Rosaceae }\end{array}$ & $\begin{array}{l}\text { Peltophorum dubium } \\
\text { (Spreng.) Taub. } \\
\text { Pyrus communis L. }\end{array}$ & $\begin{array}{l}\text { Canafistula } \\
\text { Pêra }\end{array}$ & 01 & 0,03 \\
\hline Theaceae & Camellia japonica Maquino. & Camélia & 01 & 0,03 \\
\hline Bignoniaceae & Tabebuia róseo-alba (Ridl.) & Ipê branco & 01 & 0,03 \\
\hline Apocynaceae & Plumeria speciosa Müll. Arg. & $\begin{array}{l}\text { Jasmim- } \\
\text { manga }\end{array}$ & 01 & 0,03 \\
\hline $\begin{array}{l}\text { Não identificada } \\
1\end{array}$ & & & 01 & 0,03 \\
\hline TOTAL & & & 3403 & 100,00 \\
\hline
\end{tabular}

Este percentual mostrou tendência a ampliar-se, visto que a espécie continua a ser plantada, como pode ser verificado na Figura 1, o que não é recomendável, tanto por aspectos de diversificação, quanto de harmonização e estética do ambiente. 


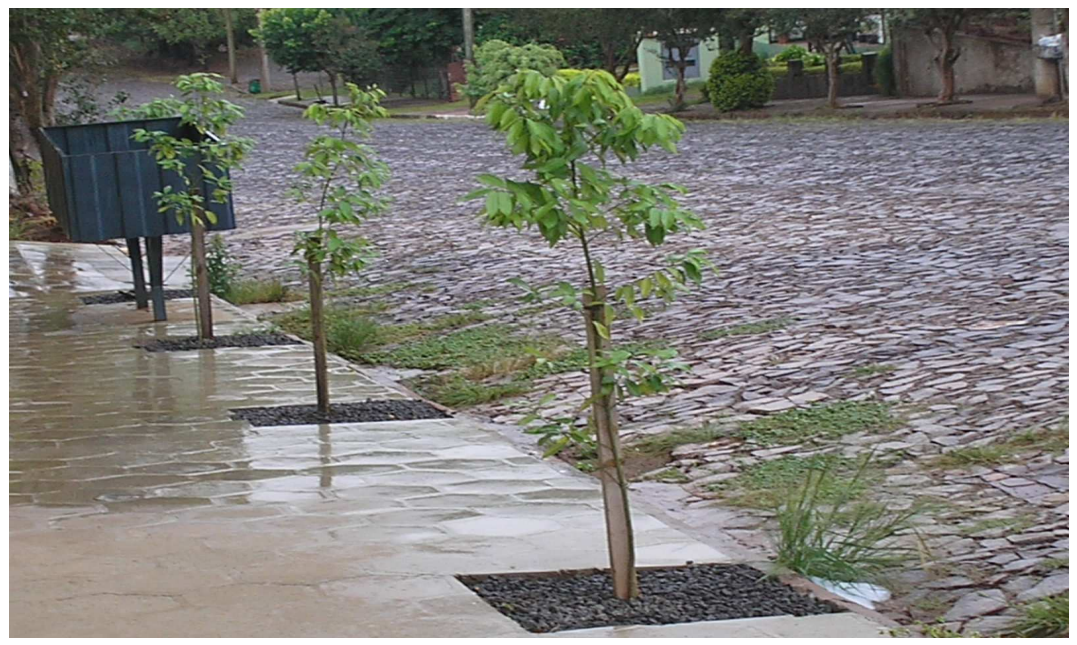

FIGURA 1 - Mudas de Cinnamomum zeylanicum L..

FIGURE 1 - Seedlings of Cinnamomum zeylanicum L..

De acordo com as recomendações de Grey e Deneke (1986), para haver um bom planejamento de arborização, cada espécie não deve ultrapassar 15\% do total de indivíduos da população arbórea, pelo fato de que a predominância de apenas uma espécie ou grupo de espécies pode facilitar a propagação das pragas, atualmente muito comum nas árvores em ambiente urbano.

A segunda espécie que apresentou maior número de indivíduos foi a Lagerstroemia indica Blanco, árvore bastante comum na arborização urbana do Estado (Figura 2), com $17,84 \%$ do total avaliado, seguida da espécie Ligustrum japonicum Thunb (8,64\%), com muitos indivíduos doentes, e a também exótica Murraya Paniculata Jack, com 6,41\%. 


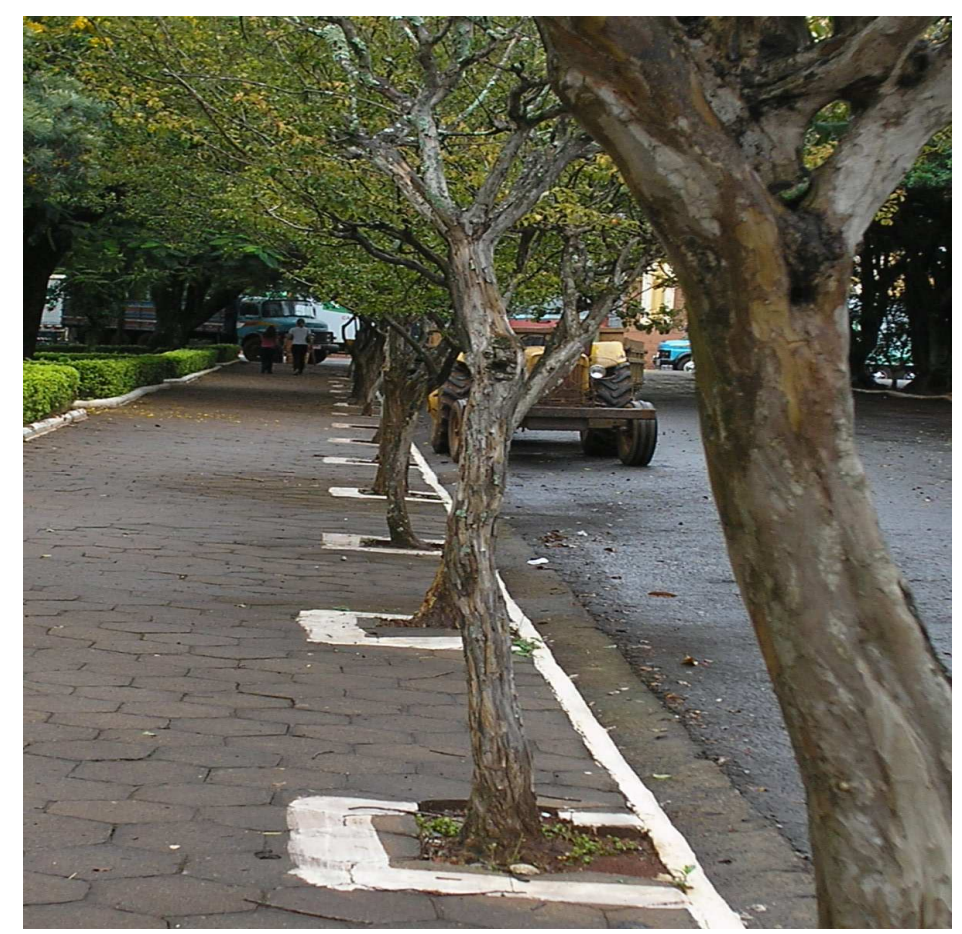

FIGURA 2 - Indivíduos da espécie Lagerstroemia indica Blanco.

FIGURE 2 - Individuals of species Lagerstroemia indica Blanco.

Importante destacar que essas quatro espécies compõem a arborização da área central do município, representando $62 \%$ da mesma, são exóticas à flora brasileira. Este aspecto está diretamente ligado à disponibilidade de espécies encontradas nas floriculturas e viveiros para venda, que, via de regra, resume-se àquelas de reprodução mais fácil e barata. Segundo Nunes (1992), é fundamental que a arborização esteja distribuída de forma diversificada pela cidade, visando uma maior eficiência ecológica e sócio-econômica.

De acordo com Detzel (1992), a arborização das cidades realizada de forma planejada, apresenta-se como uma opção para a melhoria da qualidade ambiental urbana tendo em vista os benefícios diretos e indiretos pelas árvores de rua.

A ocorrência de 451 exemplares de árvores frutíferas é outro aspecto que merece registro. Tal situação é decorrente dos plantios realizados voluntariamente pela população. $O$ plantio de frutíferas para alimentação humana não é recomendado em áreas centrais, pois a queda dos frutos pode ocasionar acidentes. 
O plantio voluntário pode levar ao excesso de espécies, gerando composições pouco estáticas e em alguns casos, a escassez de espécies pode sugerir uma monotonia, induzindo de forma imediata intervenções para adequação e ser comprometida facilmente por problemas sanitários. Deve-se buscar a diversidade de forma harmônica e na maioria das dos casos, não existe nenhum conhecimento sobre a questão, o que acaba resultando em surpresas futuras (SANTOS e TEIXEIRA, 2001).

No levantamento, a presença de frutíferas nativas, como Psidium guajava L. Campomanesia xanthocarpa Berg, Eugenia uniflora L., é interessante e deve ser estimulada, uma vez que permite a atração de espécies da fauna nativa, além de valorizar a fauna local.

No que se refere às condições fitossanitárias, encontra-se uma predominância de indivíduos danificados (79,43\%), sendo que apenas 17,57\% apresentam-se com boa integridade física e 3\% mortos (Figura 3). O alto índice de indivíduos com problemas fitossanitários, como presença de cupins, brocas, outros tipos de patógenos, injúrias físicas como anelamentos, caules ocos, podres e galhos lascados é decorrente da má utilização da poda, da evidente concentração de espécies exóticas e da falta de diversidade entre elas.

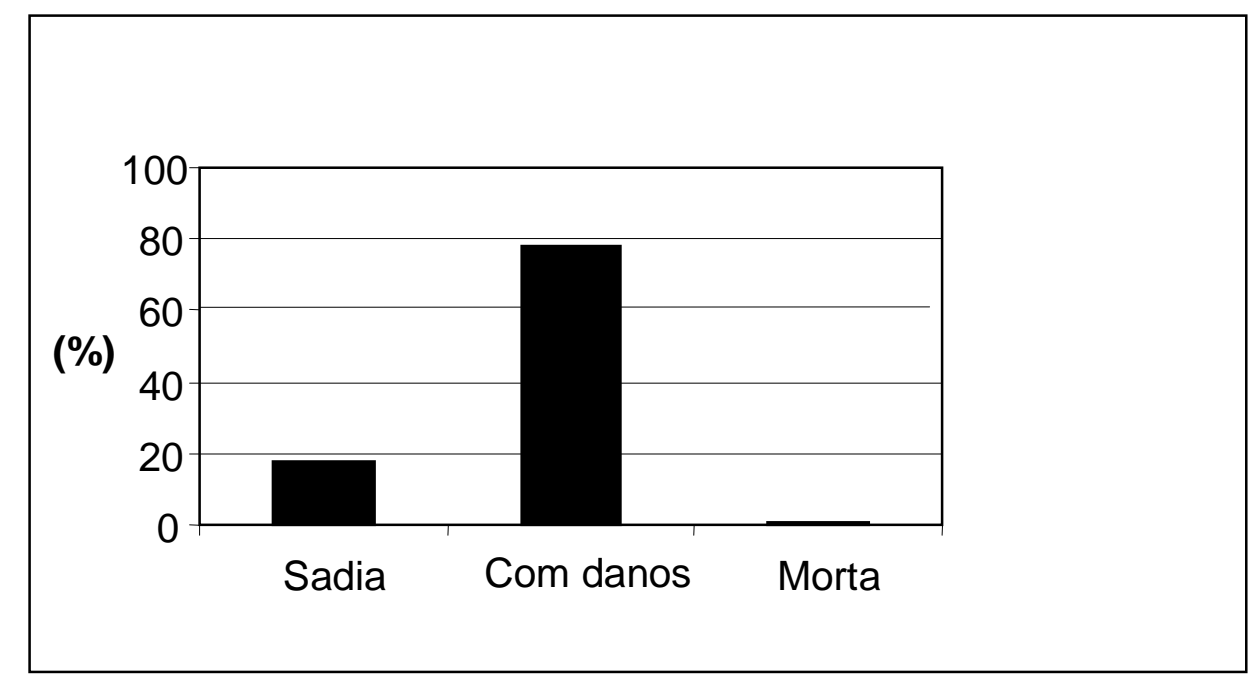

FIGURA 3 - Estado de fitossanidade das árvores avaliadas.

FIGURE 3 - Phytosanity condition of assessed trees.

No entanto, muitos são os problemas causados do confronto de árvores inadequadas com equipamentos urbanos, fiações elétricas, encanamentos, calhas, calçamentos (Figura 4), 
muros, postes de iluminação, etc. Esses problemas são comuns de serem visualizados e provocam, na maioria das vezes, um manejo inadequado e prejudicial à árvore (GUZZO, 1993).

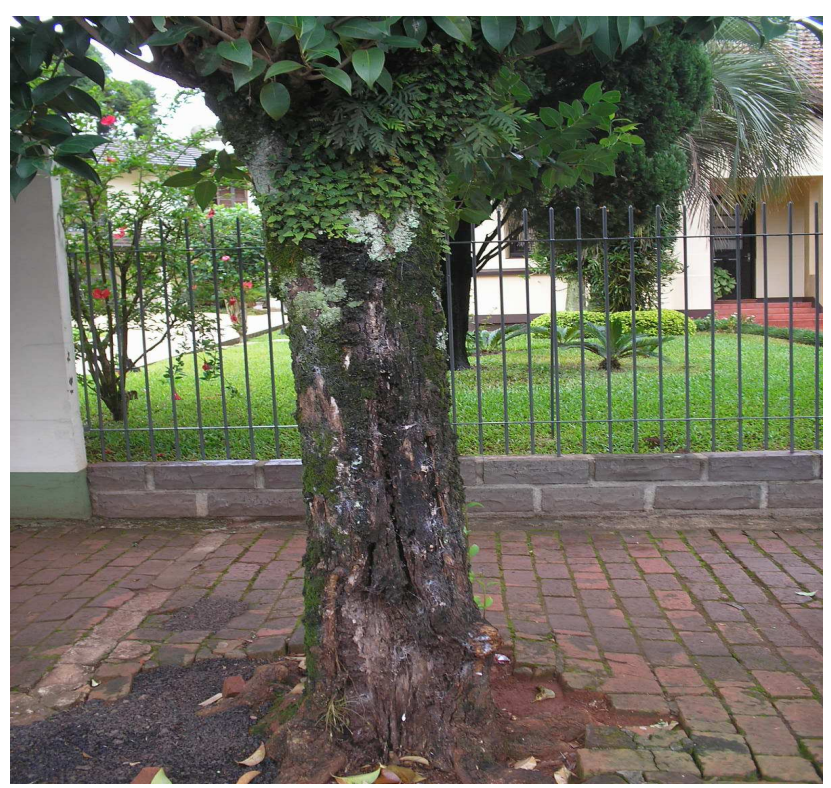

FIGURA 4 - Estado da árvore.

FIGURE 4 - Tree condition.

Em relação à distribuição da altura, $58,81 \%$ dos indivíduos abordados no estudo possui de 2 a 5 metros de altura, 21,69\% apresentam de 5 a 8 metros de altura, 11,75\% corresponderam a uma altura maior de 8 metros de altura e $7,76 \%$ são os indivíduos com altura inferior a 2 metros, considerado mudas. Esses dados estão relacionados com o CAP e o número de indivíduos cultivados na área central da cidade, conforme a tabela 2.

TABELA 2 - Classes de DAP.

TABLE 2 - Classes of DBH.

\begin{tabular}{|c|c|c|}
\hline CLASSES DE DAP $(\mathrm{cm})$ & Número de ocorrências & Freqüência (\%) \\
\hline$>5$ & 92 & 2,70 \\
\hline $5-15$ & 1349 & 39,64 \\
\hline $15-30$ & 962 & 28,27 \\
\hline $30-45$ & 346 & 10,17 \\
\hline
\end{tabular}

$\mathbf{S} \cdot \mathbf{B} \cdot \mathbf{A} \cdot \mathbf{U}$ Soc. Bras. de Arborização Urbana

REVSBAU, Piracicaba - SP, v.6, n.1, p.69-84 2011 
TABELA 2 - (continuação) Classes de DAP.

TABLE 2 - (continuation) Classes of DBH.

\begin{tabular}{|c|c|c|}
\hline $45-60$ & 104 & 3,06 \\
\hline$<60$ & 550 & 16,17 \\
\hline TOTAL & 3403 & 100 \\
\hline
\end{tabular}

Quanto à situação das raízes dos indivíduos, contatou-se que uma boa parte dos indivíduos $(53,27 \%)$ apresenta condições saudáveis, mas com grande probabilidade de futuros estragos e 32,30\% estão começando a apresentar problemas, pois estão tomando conta das calçadas, em razão de serem espécies de grande porte, necessitando de um espaçamento maior (Figura 5). Esta informação guarda estreita relação com o espaçamento disponível para as árvores, sendo que apenas $1,12 \%$ ficaram abaixo do espaçamento livre adequado, segundo Manual de Arborização da CEMIG (2001), que é de 2 metros de largura. Já os 14,43\% restantes correspondem aos indivíduos de grande porte das espécies como Grevillea robusta A. Cunn. ex R. Br. e Caesalpinia peltophoroides L. apresentam rachaduras e levantamento de calçadas constatados entre as árvores.

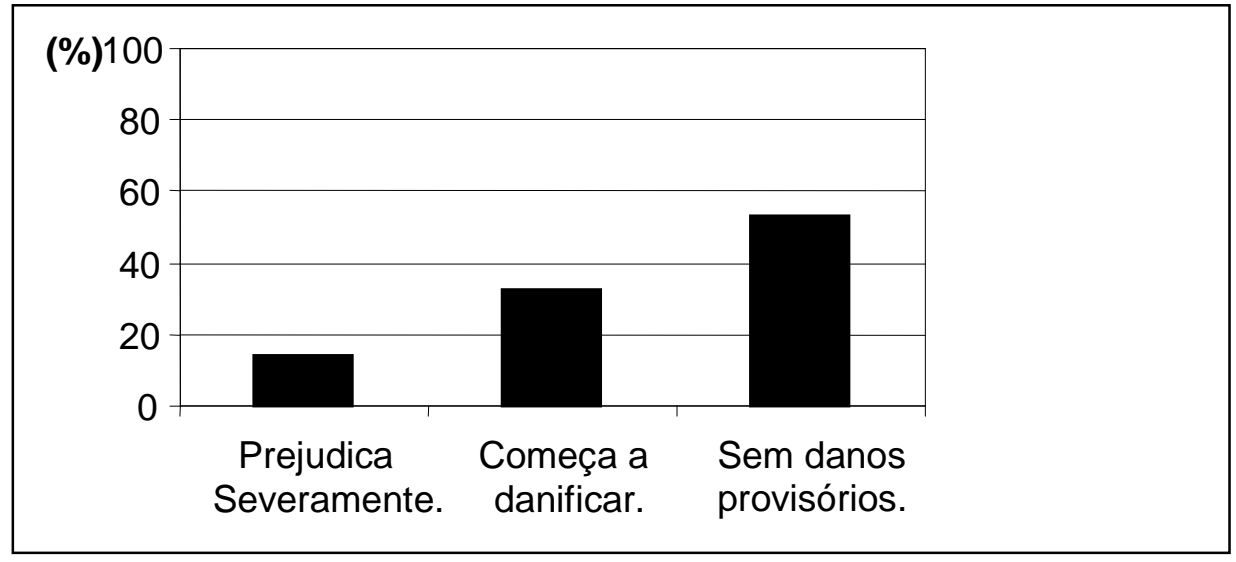

FIGURA 5 - Efeito nas calçadas ou entre espaços livres.

FIGURE 5 - Effect on sidewalks or between free spaces.

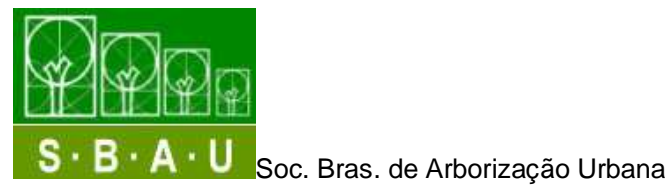

REVSBAU, Piracicaba - SP, v.6, n.1, p.69-84, 2011 
$\mathrm{Na}$ análise do espaçamento entre árvores, considerou-se como adequados os valores acima de 3 metros, que é o diâmetro de copa de uma espécie de porte pequeno. Foram constatados casos em que as árvores estavam muito próximas, como da espécie Murraya exotica L., que se encontrava em um espaçamento muito pequeno, prejudicando o trânsito dos pedestres na calçada.

TABELA 3 - Espaçamento entre árvores.

TABLE 3 - Trees spacing.

\begin{tabular}{|c|c|c|}
\hline $\begin{array}{c}\text { Espaçamento entre } \\
\text { árvores }\end{array}$ & Número de Ocorrências & Percentual (\%) \\
\hline Menor de $3 m$ & 854 & 25,12 \\
\hline Maior de $3 m$ & 2549 & 74,85 \\
\hline
\end{tabular}

As árvores podadas têm seu aspecto original alterado e jamais satisfarão as exigências impostas pela estética e ciência, embora possam satisfazer às exigências que lhes são feitas pela salubridade pública. Portanto, é bom que tenhamos árvores com seu porte natural, e para tê-las, é necessário que Ihes proporcionemos o espaçamento adequado de acordo com sua natureza. (HOEHNE,1944). A realização da poda na grande maioria dos indivíduos pesquisados $(94,87 \%)$ foi de condução. $O$ ato de podar as árvores anualmente faz parte da rotina dos moradores do sul do país, sendo que na cidade de Santo Cristo essa poda é realizada pela Prefeitura em conjunto com a RGE. Dada à abrangência de tal prática pode se deduzir ser um problema cultural, visto que nem todas as espécies requerem ou aceitam essa prática (Figura 6). Cabe ressaltar que o levantamento foi realizado no período do verão e as podas são geralmente executadas no período de inverno. 


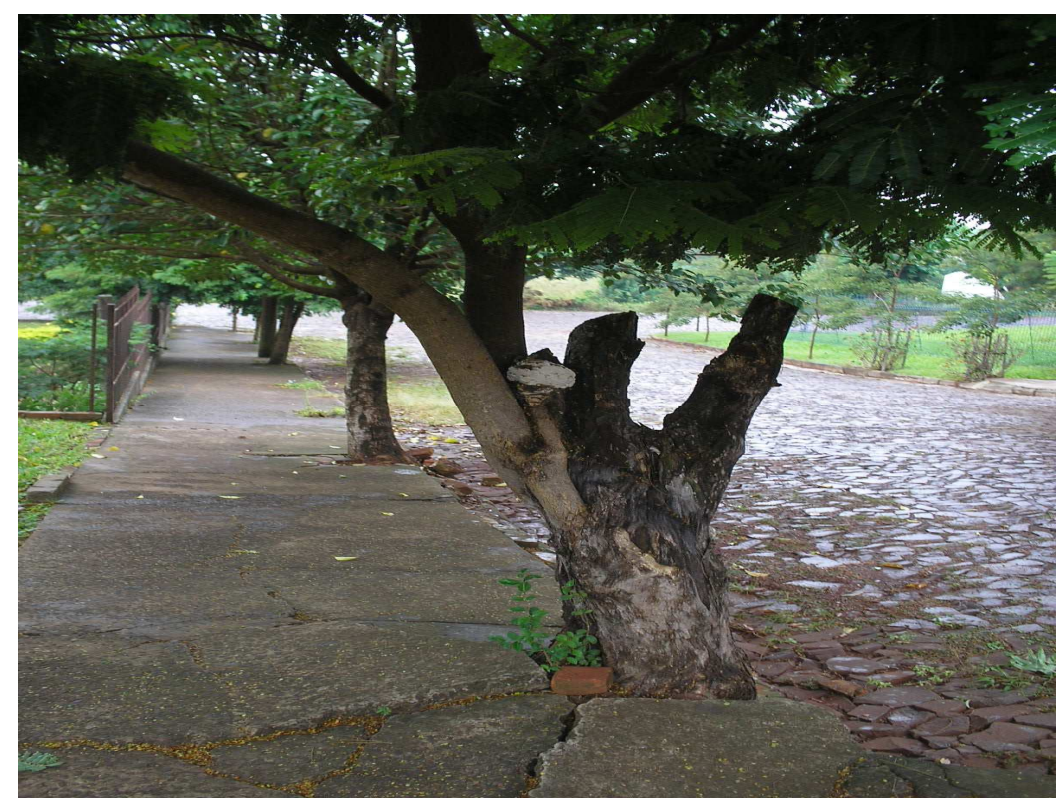

FIGURA 6 - Poda drástica e espaçamento inadequado.

FIGURE 6 - Drastic pruning and improper spacing.

\section{CONCLUSÕES}

O levantamento realizado em uma área central de Santo Cristo demonstrou que existe uma considerável quantidade de indivíduos na arborização, mas com pouca diversidade visto que 30 \% da arborização correspondeu a uma única espécie - Cinnamomum zeylanicum L. e o predomínio de espécies exóticas foi bem significativo na composição da arborização,o que não é recomendado.

As condições fitossanitárias dos indivíduos pesquisados apresentaram-se de maneira geral danificadas, em conseqüência da baixa diversidade, da falta de manejo adequado relacionado principalmente com a prática de poda inadequada e o plantio voluntário da população.

É indispensável pensar a arborização da cidade neste momento, fazendo um planejamento respeitando as limitações de cada espécie e dando preferências ás nativas, a fim de evitar mais conflitos e usufruir dos benefícios que a arborização pode representar no ambiente urbano.

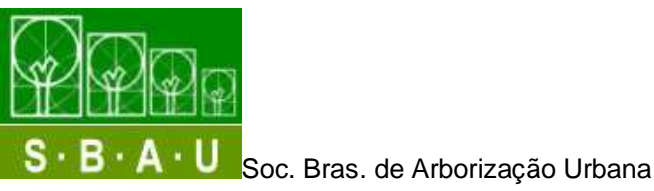




\section{AGRADECIMENTOS}

À acadêmica Elizete Patrícia Colleto, pela ajuda prestada;

À Prefeitura Municipal de Santo Cristo pelo fornecimento dos mapas e informações adicionais.

\section{REFERÊNCIAS BIBLIOGRÁFICAS}

BACKES, P.; IRGANG B. Guia de Identificação e Interesse Ecológico as Principais Espécies Nativas Sul Brasileiras. Porto Alegre: Instituto Souza Cruz, 2002. 321p.

CEMIG - COMPANHIA ENERGÉTICA DE MINAS GERAIS. Manual de arborização. Belo Horizonte: Superintendência do Meio Ambiente/CEMIG, 2001. 40p.

DETZEL,V. A. . A cidade, os espaços e a vegetação In: CONGRESSO BRASILEIRO SOBRE ARBORIZAÇÃO URBANA,1; ENCONTRO NACIONAL SOBRE ARBORIZAÇÃO URBANA,4.1992,VITÓRIA. Anais...Vitória: SBAU, 1992. p.39-51.

GREY,G. W., DENEKE, F. J. Urban forestry. New York: John Wiley \& Sons, 1986. 279 p.

GUZZO, P. Alterações Ambientais em Áreas Urbanas: planejamento e legislação ambiental. In: SEMINÁRIO LATINO AMERICANO DE PLANEJAMENTO URBANO, 1993, Campo Grande,MS. Anais..., Campo Grande: UFMT, 1993. p.214-222.

HOEHNE, F. C. Arborização urbana. São Paulo: Secretaria de Agricultura, Indústria e Comércio,1944.215p.

IBGE - INSTITUTO BRASILEIRO DE GEOGRAFIA E ESTATÍSTICA. Disponível em: <http://www.ibge.gov.br/cidadesat/default.php>. Acesso em 27 set. 2006.

MARTINS JÚNIOR, O. P. Uma Cidade Ecologicamente Correta. Goiânia: a.b., 1996.

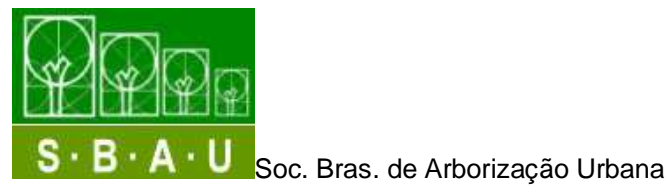


MILANO, M. S. Avaliação e análise da arborização de ruas de Curitiba-PR. Curitiba,UFPR: Curso de Pós-Graduação em Engenharia Florestal,1984.130p. (Dissertação de Mestrado).

NUNES, M. L. A Cidade, os Espaços Abertos e Vegetação. In: CONGRESSO BRASILEIRO SOBRE ARBORIZAÇÃO URBANA,1; ENCONTRO NACIONAL SOBRE ARBORIZAÇÃO URBANA,4.1992,VITÓRIA. Anais...Vitória: SBAU, 1992. p.133-145.

SANTOS, N.R.Z.; TEIXEIRA, I.F. Arborização de Vias Públicas:Ambiente X Vegetação. Santa Cruz do Sul, RS: Instituto Souza Cruz, 2001. 135p. 